\title{
Classic Kaposi Sarcoma: to treat or not to treat?
}

\author{
Bruno Vincenzi ${ }^{1}$, Loretta D'Onofrio ${ }^{1}$, Anna Maria Frezza ${ }^{1 *}$, Rosario Francesco Grasso ${ }^{2}$, Valentina Fausti ${ }^{1}$, \\ Daniele Santini ${ }^{1}$, Angelo Paolo Dei Tos $^{3}$ and Giuseppe Tonini ${ }^{1}$
}

\begin{abstract}
Background: Classic Kaposi Sarcoma (KS) is vascular sarcoma, known to be more common in Mediterranean elderly men and characterized by an indolent clinical behavior. To our knowledge, this is the first evidence in literature, describing a spontaneous partial regression in a non-HIV, non-iatrogenic KS.

Case presentation: A 68-years old woman, presenting with weight loss and respiratory symptoms, was diagnosed with a classic KS involving lungs and mediastinal lymph nodes. No skin or mucosal lesions were identified, HIV positivity was ruled out. Due to patient's choice, she was kept under surveillance with 3-monthly thorax-abdomen-pelvis computed tomography scan (TAP CT). A first reassessment proved progressive disease (PD) associated with symptoms worsening. A new TAP CT, performed at 5 months from the diagnosis, showed stable disease (SD), with a minor reduction in size of mediastinal lymphadenopathies. A further reassessment, performed 5 months later, resulted in a partial response (PR) despite the absence of any medical treatment. Up to date, the disease is in remission, patient is asymptomatic and still on surveillance.
\end{abstract}

Conclusion: Given the possible indolent behaviour of KS, we believe that close surveillance can represent a valuable approach in selected cases.

Keywords: Classic Kaposi sarcoma, Spontaneous regression

\section{Background}

KS is a locally aggressive endothelial tumour belonging to the family of vascular sarcoma. According to aetiology and epidemiology, four variants have been described: classic KS, usually affecting Mediterranean elderly men; endemic African KS, common in middle-aged adults and children in Equatorial Africa; iatrogenic KS, usually occurring in solid organ transplant recipients but also in patients receiving immunosuppressive treatment (i.e. corticosteroid) for a long time; acquired immunodeficiency syndrome associated KS, the most aggressive variant, affecting human immunodeficiency virus (HIV) positive patients. KS is invariably associated with human herpes virus type 8 (HHV8), which seems to play a key role in KS pathogenesis [1]. KS typically presents with mucocutaneous lesions, mostly affecting lower extremities, face, trunk, genitalia and oropharyngeal mucosa, but it can also involve lymph nodes and visceral organs, including the respiratory and

\footnotetext{
* Correspondence: a.frezza@unicampus.it

'Department of Medical Oncology, University Campus Bio-Medico, via Alvaro del Portillo 21, Rome, Italy

Full list of author information is available at the end of the article
}

gastrointestinal tracts [2]. Clinical behaviour is often indolent, especially in the classic variant.

Pulmonary involvement is common in critically immunodeficient patients, occurring approximately in $45 \%$ of those with cutaneous AIDS-related KS with previous or concomitant mucocutaneous lesions. Lung metastases from sporadic KS are rare, especially in female and pediatric series with a distinct male predominance [3]. Treatment options for non-HIV forms include chemotherapy (liposomal doxorubicin, vinblastine, taxanes) [4], immunotherapy (interferon alpha, interleukin-12) and anti-HHV8 therapy [5]. Close surveillance can also be a possibility in selected cases.

\section{Case presentation}

A 68-years old woman, with no previous medical history, presented with severe weight loss, shortness of breath and cough (ECOG PS: 2). A chest $x$-ray followed by a thorax-abdomen-pelvis computed tomography (TAP CT) showed the presence of a $53 \mathrm{~mm}$ large mass in the left lower lobe (LLL) with pericardial involvment and a $30 \times 18 \mathrm{~mm}$ large lesion with similar morphological features in the upper lobe of the left lung. Multiple bilateral 


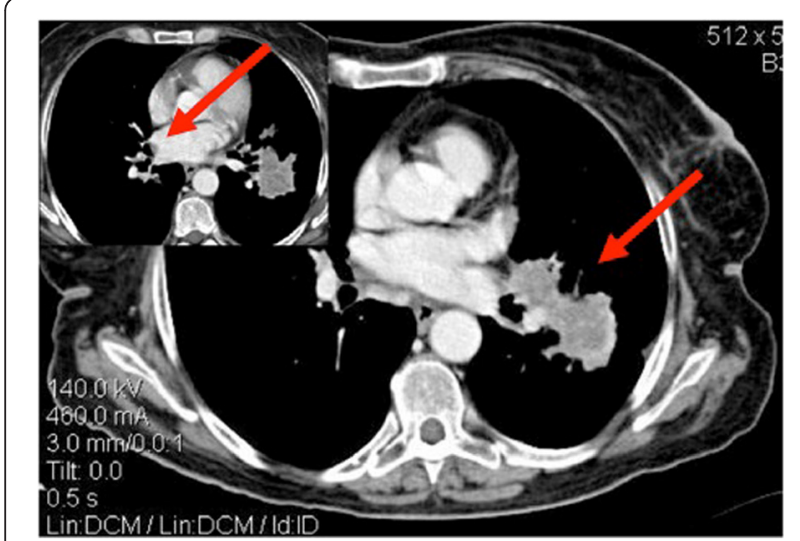

Figure 1 Baseline thorax-abdomen-pelvis computed tomography, showing a large mass in the left lower lobe, multiple bilateral pulmonary nodules and mediastinal adenophaties.

lung nodules and a $26 \mathrm{~mm}$ large carinal lymphadenopathy were also reported (Figure 1). Bronchoscopic and CTguided biopsies were attempted without success, ending up with a surgical exploration and complete removal of one lung nodule. Pathology proved a malignant stromal tumor with endothelial phenotype, consistent with a pulmonary localization of KS. Immunohistochemistry resulted positive for CD31, CD 34 and HHV-8; Ki67 10\% (Figure 2). Physical examination ruled out any suspicious skin lesions, while upper gastrointestinal endoscopy and colonoscopy excluded mucosal localizations. Anti-HIV antibody and HIV antigen tests were both negative.
Given patient's refusal of any medical treatment, she was started on a surveillance program. The first reassessment proved PD according to RECIST 1.1 criteria, with the appearance of a new nodule in the right mid lobe (RML), an increase in size of the known lesion in the LLL $(60 \mathrm{~mm})$ and a stability of the carinal lymphnodes (Figure 3). Despite the persistence of symptoms, due to patient's choice, no treatment was started. A new radiological assessment, performed after 5 months from diagnosis, showed a minor reduction in size of the lymphadenopathies and an almost complete resolution of the nodule in the RML. Given radiological evidence of disease regression and improvement in symptoms, the patient underwent a further TAP CT five months later, showing PR, with a reduction in number of lung nodules and reduction in size on both the lesion in the LLL and carinal lymphnodes (Figure 4). Up to date, at nineteen months from diagnosis, disease is still in remission with stable pulmonary nodules and no evidence of systemic spread, in absence of any medical treatment. The patient is still on surveillance through three-monthly clinical assessment and six-monthly TAP CT, asymptomatic, ECOG PS: 0 .

\section{Conclusion}

Spontaneous regression of KS has been previously described in the iatrogenic type. Interruption of corticosteroids [6], systemic immunosuppressive drugs $[7,8]$, and ACE-inhibitors [9] can lead to a spontaneous selfhealing of KS skin lesions. In AIDS-related subtype,



Figure 2 Histology and immunohistochemistry (CD31, CD34 and HHV-8). 


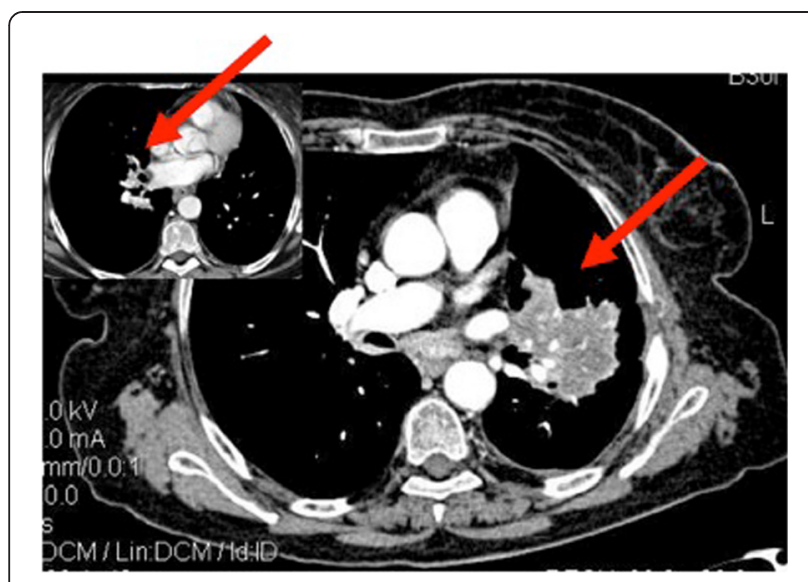

Figure 3 Thorax-abdomen-pelvis computed tomography two months after diagnosis, showing progressive disease.

disease regression usually results from intensive antiretroviral therapy $[10,11]$. To our knowledge, this is the first evidence in literature, describing a spontaneous partial regression in non-HIV, non-iatrogenic Kaposi sarcoma. In Kondo's paper [12], multiple skin lesion histologically suspected for sporadic Kaposi sarcoma, disappeared after the discontinuation of a prolonged steroid therapy administered for chronic respiratory failure. In the present case report, no trigger mechanisms leading to the development of this disease were identified, nor removable stimuli to malignant proliferation. There are no data in literature providing evidence for possible biological mechanism underlying this spontaneous regression of disease. Conversely, the presence of herpes virus infection and any concomitant changes in the balance of immune system are well recognized factors promoting the development of this cancer [13-16]. Therefore, we might assume that a fully competent immune system, in absence of the impairment induced by chemotherapy administration, could have played a role in the observed

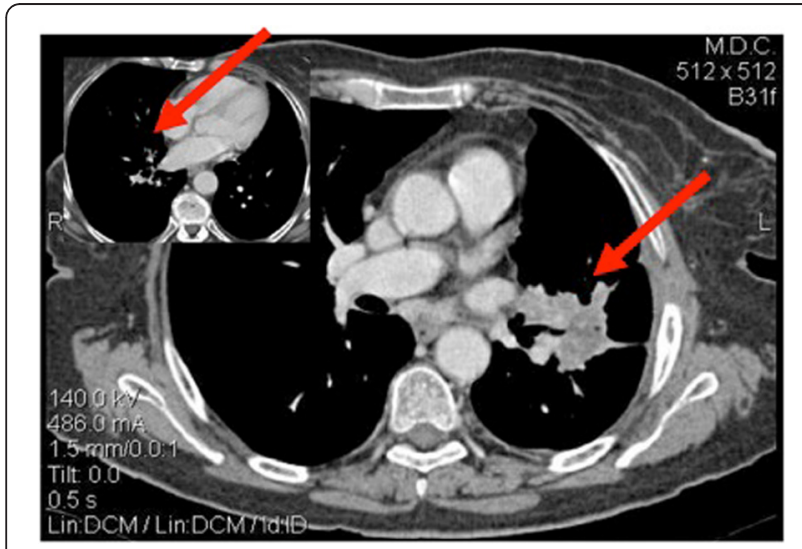

Figure 4 Thorax-abdomen-pelvis computed tomography two months after diagnosis, showing partial response. disease regression. However, future studies are needed to verify the reliability of this hypothesis and, despite this interesting observation, more data are needed to support close surveillance as a management strategy in this rare disease.

\section{Consent}

Written informed consent was obtained from the patient for publication of this Case Report and any accompanying images. A copy of the written consent is available for review by the Editor-in-Chief of this journal.

\section{Abbreviation}

KS: Kaposi sarcoma; LLL: Left lower lobe; PD: Progressive disease according to RECIST 1.1 criteria; PR: Partial response according to RECIST 1.1 criteria; RML: Right mid lobe; SD: Stable disease according to RECIST 1.1 criteria; TAP TC: Thorax-abdomen-pelvis computed tomography scan.

\section{Competing interests}

The authors declare that they have no competing interests.

\section{Authors' contributions}

BV, DS, GT conceived the study, and participated in its design. APDT, RFG collected the data. AMF, LD, VF helped to collect the data and draft the manuscript. All authors read and approved the final manuscript.

\section{Author details}

${ }^{1}$ Department of Medical Oncology, University Campus Bio-Medico, via Alvaro del Portillo 21, Rome, Italy. ${ }^{2}$ Department of Radiology, University Campus Bio-Medico, via Alvaro del Portillo 21, Rome, Italy. ${ }^{3}$ Department of Pathology, General Hospital, P.zza Ospedale 1, Treviso, Italy.

Received: 19 December 2013 Accepted: 19 March 2015 Published online: 10 April 2015

\section{References}

1. Moore PS, Chang Y. Detection of herpesvirus-like DNA sequences in Kaposi's sarcoma in patients with and without HIV infection. N Engl J Med. 1995;332:1181-5

2. Antman K, Chang Y. Kaposi's sarcoma. N Engl J Med. 2000;342:1027-38.

3. Godoy MC, Rouse H, Brown JA, Phillips P, Forrest DM, Müller NL. Imaging features of pulmonary Kaposi sarcoma-associated immune reconstitution syndrome. Am J Roentgenol. 2007;189:956-65.

4. Régnier-Rosencher E, Guillot B, Dupin N. Treatments for classic Kaposi sarcoma: a systematic review of the literature. J Am Acad Dermatol. 2013;68:313-31.

5. Akasbi Y, Awada A, Arifi S, Mellas N, El Mesbahi O. Non-HIV Kaposi's sarcoma: a review and therapeutic perspectives. Bull Cancer. 2012;99:92-9.

6. Tebbe B, Mayer-da-Silva A, Garbe C, Von Keyserlingk HJ, Orfanos CE. Genetically determined coincidence of Kaposi sarcoma and psoriasis in an HIV-negative patient after prednisolone treatment: spontaneous regression 8 months after discontinuing therapy. Int J Dermatol. 1991;30:114-20.

7. Hussein MM, Mooij JM, Roujouleh HM. Regression of posttransplant Kaposi sarcoma after discontinuing cyclosporin and giving mycophenolate mofetil instead. Nephrol Dial Transplant. 2000;15:1103-4.

8. Nagy S, Gyulai R, Kemeny L, Szenohradszky P, Dobozy A. latrogenic Kaposi's sarcoma: HHV8 positivity persists but the tumors regress almost completely without immunosuppressive therapy. Transplantation. 2000;69:2230-1.

9. Dervis E, Demirkesen C. Kaposi's sarcoma in a patient with psoriasis vulgaris. Acta Dermatovenerol Alp Panonica Adriat. 2010;19:31-4.

10. Real FX, Krown SE. Spontaneous regression of Kaposi's sarcoma in patients with AIDS. N Engl J Med. 1985;313:1659.

11. Aboulafia DM. Regression of acquired immunodeficiency syndrome-related pulmonary Kaposi's sarcoma after highly active antiretroviral therapy. Mayo Clin Proc. 1998;73:439-43.

12. Kondo Y, Izumi T, Yanagawa T, Kanda H, Katano H, Sata T. Spontaneously regressed Kaposi's sarcoma and human herpesvirus 8 infection in a human immunodeficiency virus-negative patient. Pathol Int. 2000;50:340-6. 
13. Douglas JL, Gustin JK, Dezube B, Pantanowitz UL, Moses AV. Kaposi's sarcoma: a model of both malignancy and chronic inflammation. Panminerva Med. 2007:49(3):119-38.

14. Brown EE, Whitby D, Vitale F, Marshall V, Mbisa G, Gamache C, et al. Virologic, hematologic, and immunologic risk factors for classic Kaposi sarcoma. Cancer. 2006;107(9):2282-90.

15. Sung JC, Louie SG, Park SY. Kaposi's sarcoma: advances in tumor biology and pharmacotherapy. Pharmacotherapy. 1997;17(4):670-83.

16. Galleu A, Fozza C, Simula MP, Contini S, Virdis P, Corda G, et al. CD4 ${ }^{+}$and $\mathrm{CD}^{+} \mathrm{T}$-Cell Skewness in classic Kaposi Sarcoma. Neoplasia. 2012;14(6):487-94

Submit your next manuscript to BioMed Central and take full advantage of:

- Convenient online submission

- Thorough peer review

- No space constraints or color figure charges

- Immediate publication on acceptance

- Inclusion in PubMed, CAS, Scopus and Google Scholar

- Research which is freely available for redistribution 